$1 N \cdot 34$

NASA Technical Memorandum 109104
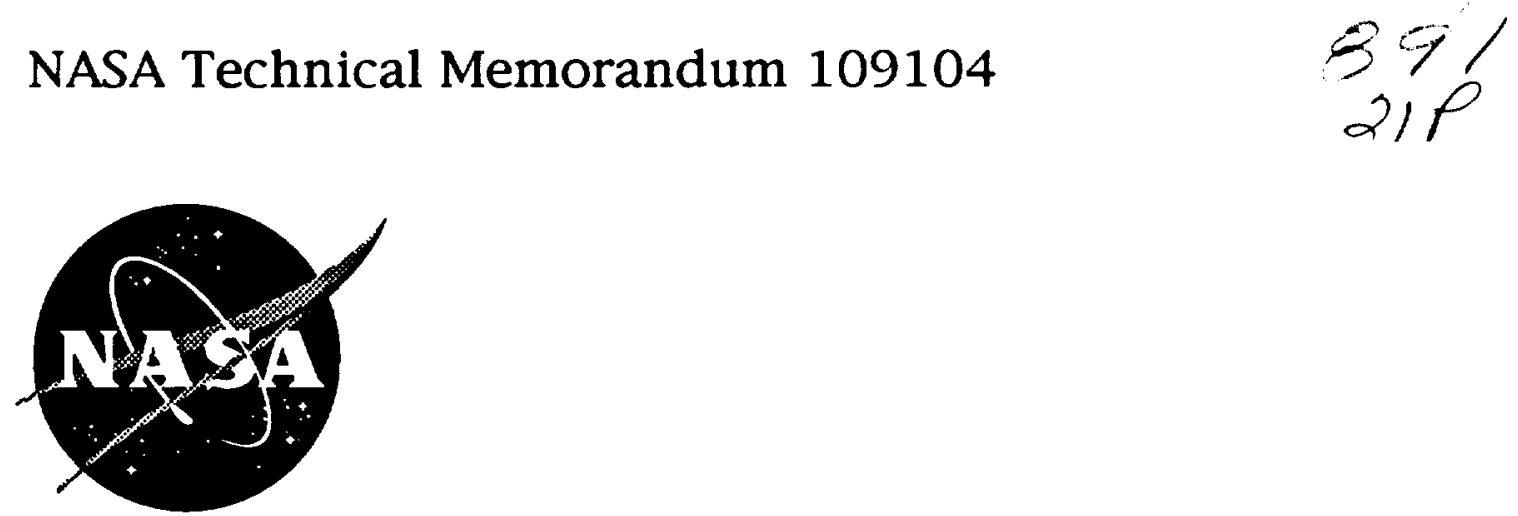

\title{
NEW CONSTITUTIVE EQUATION FOR THE VOLUME VISCOSITY IN FLUIDS
}

Allan J. Zuckerwar

Langley Research Center, Hampton, Virginia

Robert L. Ash

Old Dominion University, Norfolk, Virginia

March 1994

National Aeronautics and

Space Administration

Langley Research Center

Hampton, Virginia 23681-0001

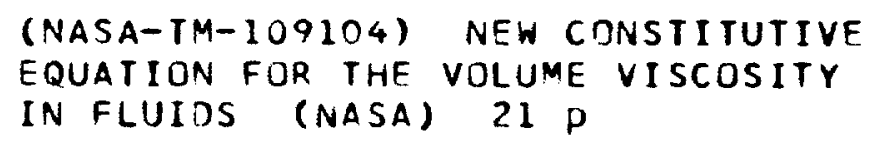




\section{Introduction}

The traditional stress tensor for a viscous fluid

$$
\sigma_{i j}=-\left[p-\lambda \frac{\partial u_{m}}{\partial x_{m}}\right) \delta_{i j}+\mu\left(\frac{\partial u_{i}}{\partial x_{j}}+\frac{\partial u_{j}}{\partial x_{i}}\right)
$$

comprises terms representing the thermodynamic pressure, the volume viscosity, and the shear viscosity. Unlike the shear viscosity $\mu$, which can be derived from the Boltzmann Transport Equation and thus lends itself to a microscopic interpretation [1], the traditional volume viscosity $\lambda$ has no physical basis and no relationship to established dissipative processes in fluids. In other words, neither its value nor even its order of magnitude can be predicted from the fundamental physical properties of a given fluid. In order to circumvent this obstacle to an orderly analysis of viscous flows, stokes hypothesized that the volume losses in fluids are negligibly small [2] (which is true by definition for incompressible flows). This so-called "Stokes hypothesis"

$$
\lambda=-(2 / 3) \mu
$$

has been utilized in a large volume of both experimental and theoretical data in many classes of compressible convective flows.

In nonconvective (periodic or acoustic) flow, on the other hand, the point of view that volume losses are negligibly small has no basis in fact, to which the vast literature on sound absorption in fluids will attest. The underlying absorption processes in gases and some liquids are well understood and known to be attributable to a variety of relaxation processes, which accordingly constitute the physical basis for the volume viscosity. In gases the most prominent of these under ordinary experimental conditions are the relaxations of the molecular degrees of freedom.

The purpose of this memorandum is to close the longstanding gap between acoustics and fluid dynamics with regard to volumetric losses in fluids. We start out by reviewing the fundamental physics of relaxation and its mathematical representation. Then we apply the lossy Navier-stokes Equation to periodic (acoustic) flow and show that the traditional 
expression for volume viscosity leads to a result which contradicts that describing acoustical relaxation. We demonstrate that the addition of a second volume viscosity term resolves the conflict and leads to a direct correspondence between the volume viscosity and the acoustic relaxation parameters. We proceed to discuss the formulation of volume viscosity in the presence of multiple relaxations. Finally, we present an example of the role of volume viscosity in a convective compressible flow. Although our discussion will be confined to gases, our analysis and conclusions will apply equally well to liquids since the mathematical representation of relaxation is the same in both media.

\section{8ymbols}

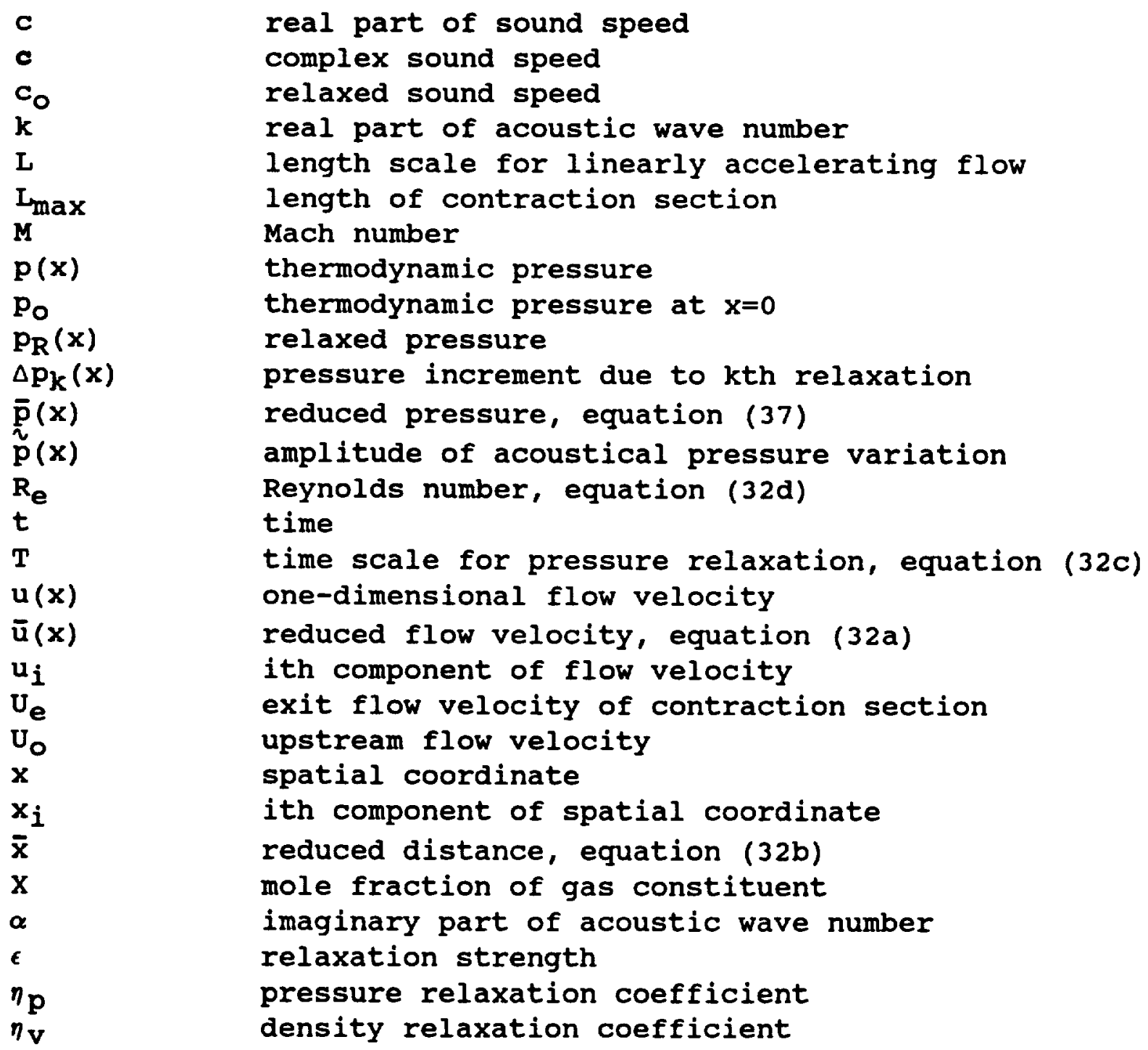




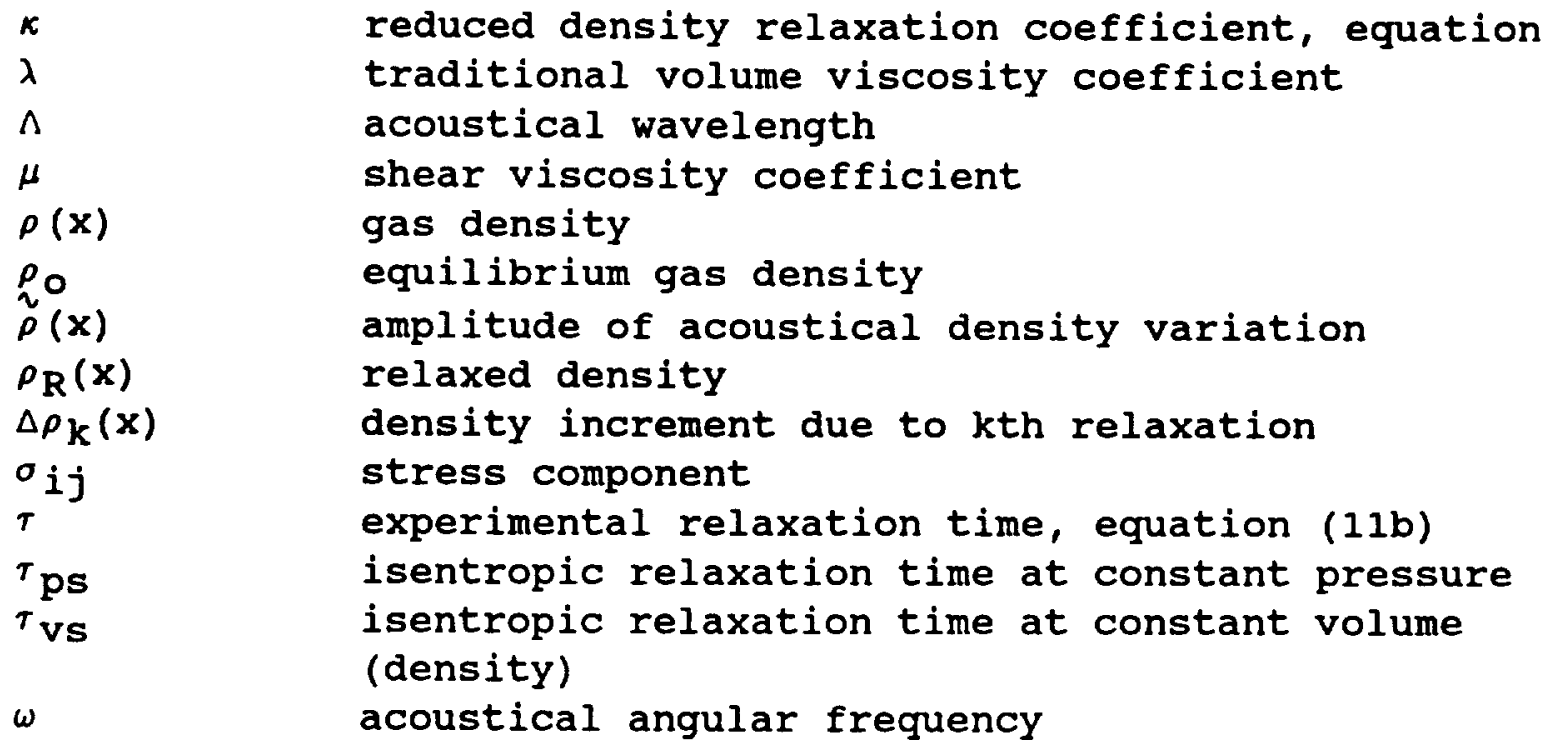

\section{Numerical subscripts}

$$
\begin{array}{ll}
i, j, m & \text { indicates stress component }(i, j, m=1,2,3) \\
k & \text { indicates relaxation process }(k=1,2, \ldots)
\end{array}
$$

Mathematical operators

$$
\begin{array}{ll}
\mathrm{D} / \mathrm{Dt} & =a / \partial t+u \partial / \partial \mathrm{x}, \text { one-dimensional total time } \\
& \text { derivative } \\
\delta_{i j} & =0 \text { if } i \neq j,=1 \text { if } i=j, \text { Kroniker delta } \\
0(j) & \text { indicates order of magnitude }
\end{array}
$$

\section{Acoustic Relaxation in Gases}

The following derivation of the acoustic relaxation equations is based on small periodic (harmonic) variations, one-dimensional propagation, and a single relaxation process. The equations of motion

$$
\rho_{\circ} \frac{\partial u}{\partial t}=-\frac{\partial p}{\partial x}
$$

and continuity

$$
\frac{\partial u}{\partial x}=-\frac{1}{\rho_{0}} \frac{\partial \rho}{\partial t}
$$

lead to a quasi-wave equation 


$$
\frac{\partial^{2} \rho}{\partial t^{2}}=\frac{\partial^{2} p}{\partial x^{2}}
$$

which is satisfied by travelling wave solutions of the form

$$
\begin{aligned}
& \rho=\tilde{\rho} e^{i(\omega t-k x)} \\
& p=\tilde{p} e^{i(\omega t-k x)}
\end{aligned}
$$

When we insert $(6 a)$ and $(6 b)$ into the acoustical equation of state [3]

$$
\left(1+r_{\mathrm{ps}} \frac{\partial}{\partial t}\right) \rho=\frac{1}{\mathrm{co}_{\mathrm{o}}{ }^{2}}\left(1+\tau_{\mathrm{vs}} \frac{\partial}{\partial t}\right) \mathrm{p}
$$

we obtain the ratio $\tilde{\rho} / \tilde{p}$, which is the the reciprocal complex sound speed squared:

$$
\underset{\tilde{p}}{\tilde{p}}=\frac{1}{c^{2}}=\frac{1}{c_{0}^{2}} \frac{1+i \omega \tau \mathrm{vs}}{1+i \omega \tau \mathrm{ps}}+o\left(\epsilon^{2}\right)
$$

Upon separating equation (8) into real and imaginary parts, we isolate the terms representing the sound dispersion (real) and absorption (imaginary):

$$
\frac{c_{0}^{2}}{c^{2}}=1-\epsilon \frac{\omega^{2} \tau p s^{2}}{1+\omega^{2} \tau p s^{2}}-i \epsilon \frac{\omega \tau p s}{1+\omega^{2} \tau p s^{2}}+o\left(\epsilon^{2}\right)
$$

or, equivalently

$$
\frac{c^{2}}{c_{0}^{2}}=1+\frac{\epsilon}{1-\epsilon} \frac{\omega^{2} \tau^{2}}{1+\omega^{2} \tau^{2}}+i \frac{\epsilon}{\sqrt{1-\epsilon}} \frac{\omega \tau}{1+\omega^{2} \tau^{2}}+O\left(\epsilon^{2}\right)
$$

As indicated in equations (9) and (10), a single relaxation process contains two defining parameters, a "relaxation strength" and a "relaxation time." The parameters used in phenomenological theories of relaxation are the isentropic relaxation times at constant pressure $\tau$ ps and at constant volume $\tau_{\text {vs, but those }}$ obtained from acoustical measurements are the relaxation strength $\epsilon$ and a third relaxation time $\tau$. The parameter pairs are interrelated by 


$$
\begin{aligned}
& \epsilon=\frac{{ }^{\top} \mathrm{ps}^{-\tau} \mathrm{vs}}{\tau \mathrm{ps}} \\
& \tau=\sqrt{\tau \mathrm{ps}^{\tau} \mathrm{vs}}
\end{aligned}
$$

or conversely,

$$
\begin{aligned}
& \tau_{\mathrm{ps}}=\tau / \sqrt{1-\epsilon} \\
& \tau_{\mathrm{vs}}=\tau \sqrt{1-\epsilon}
\end{aligned}
$$

The relaxation strength is a measure of the capacity of the medium to convert translational energy of the flow into internal energy. It can be evaluated with great precision from known thermodynamic properties of the medium. The relaxation time is a measure of the rapidity with which the medium reacts to changes in translational energy. Because microscopic theories of the relaxation time are generally imprecise, quantitative evaluation depends on experiment. Information on the relaxation processes occurring in air are given later in this memorandum.

It is important to note that the relaxation strength depends upon the difference between $\tau_{\text {ps }}$ and $\tau_{\text {vs }}$, a fact not taken into account in previous treatments of the volume viscosity.

The real part of equation (10) yields the dispersion of the sound speed squared, shown in the plot of figure 1 . The "dispersion step" has a height of $\epsilon /(1-\epsilon)$, equal to the difference between the low and high frequency limiting solutions of the plot, and an inflection point where the condition $\omega \tau=1$ is fulfilled.

The imaginary part of equation (10) yields the sound absorption per unit wavelength:

$$
\begin{aligned}
& \alpha \Lambda \frac{c_{0}^{2}}{c^{2}}=\frac{\pi \epsilon}{\sqrt{1-\epsilon}} \frac{\omega \tau}{1+\omega^{2} \tau^{2}} \\
& \alpha \Lambda=\frac{\pi \epsilon}{\sqrt{1-\epsilon}} \frac{\omega \tau}{1+\omega^{2} \tau^{2}}+O\left(\epsilon^{2}\right)
\end{aligned}
$$


The plot of equation (13b) in Fig. 2 shows an absorption peak of height $\pi \epsilon /(2 \sqrt{1-\epsilon})$ and a location at $\omega \tau=1$.

Both plots reveal that a relaxation process is ineffective at very low frequencies, where the internal degrees of freedom have ample time to equilibrate with translation, and at very high frequencies, where changes in translation occur too rapidly for equilibration to take place. In convective compressible flows, then, the volume viscosity would be expected to be most effective on time scales on the order of the relaxation time $\tau$.

\section{Traditional Volume Viscosity and Application to Acoustics}

We observe that the acoustical wave equation (5) does not contain a dissipative term. Dissipation is accounted for in the complex sound speed, which is derived from the acoustical equation of state (7). The introduction of a physically meaningful volume viscosity implies a transfer of the relaxation terms from the equation of state to the Navier-stokes Equation. Then, the equation of state will serve simply to determine the stationary temperature of the medium.

To investigate the case of damped acoustical propagation, let us insert the traditional stress tensor [equation (1)] into the one-dimensional Navier-stokes Equation, retaining only the dilatational terms. In place of equation (3) we obtain

$$
\rho_{\circ} \frac{\partial u}{\partial t}=\frac{\partial \sigma_{11}}{\partial x}=-\frac{\partial p}{\partial x}+\eta_{v} \frac{\partial^{2} u}{\partial x^{2}}
$$

where for one-dimensional propagation

$$
\eta_{\mathrm{v}}=\lambda+2 \mu
$$

Equation (14) together with the continuity equation (4) leads to the lossy wave equation:

$$
\frac{\partial^{2} \rho}{\partial t^{2}}-\frac{\eta_{v}}{\rho_{0}} \frac{\partial^{3} \rho}{\partial x^{2} \partial t}=\frac{\partial^{2} p}{\partial x^{2}}
$$

Solutions of the form (6a) and (6b) yield the complex sound speed: 


$$
\underset{\tilde{p}}{\tilde{p}}=\frac{1}{c^{2}}=\frac{\mathrm{k}^{2}}{\omega^{2}-i \omega\left(\eta_{\mathrm{v}} / \rho_{0}\right) \mathrm{k}^{2}}
$$

from which

$$
\frac{c_{o}^{2}}{c^{2}}=1-\frac{\omega^{2} \eta_{v}^{2} / \rho_{o}^{2} c_{o}^{4}}{1+\omega^{2} \eta_{v}^{2} / \rho_{o}^{2} c_{o}^{4}}+i \frac{\omega \eta_{v} / \rho_{o} c_{o}^{2}}{1+\omega^{2} \eta_{v}^{2} / \rho_{o}^{2} c_{o}{ }^{4}}+o\left(\epsilon^{2}\right)
$$

A term-by-term comparison between equations (18) and (9) reveals that the quantity $\eta_{v} / \rho_{o} c_{o}{ }^{2}$ can be identified with $-\tau$ ps, but that the two equations are compatible only if $\epsilon=1--a n$ absurd conclusion, for kneser proves that the relaxation strength of a diatomic gas is bounded by $\epsilon<0.082$ for the vibrational relaxation and $\epsilon<0.16$ for the rotational relaxation [4]. Therefore, we conclude that the volume viscosity cannot be represented by the traditional constitutive equation (1), containing only a single term.

\section{New Constitutive Equation for the volume viscosity}

Taking a cue from the acoustical equation of state (7), we hypothesize that the second volume viscosity term is proportional to the time derivative of the pressure. Then the stress tensor becomes

$\sigma_{i j}=-\left[p-\eta_{p} \frac{D p}{D t}-\eta_{v} \frac{\partial u_{m}}{\partial x_{m}}\right] \delta_{i j}+\mu\left(\frac{\partial u_{i}}{\partial x_{j}}+\frac{\partial u_{j}}{\partial x_{i}}-\frac{2}{3} \frac{\partial u_{m}}{\partial x_{m}} \delta_{i j}\right)$

where for one-dimensional flow the factor $2 / 3$ is replaced by 2 , and the operator

$$
\frac{D}{D t}=\frac{\partial}{\partial t}+u \frac{\partial}{\partial x}
$$

is used to allow for pressure changes in convective flow. Let us examine the acoustical consequences. When we substitute the nonconvective dilatational terms of (19) into the one-dimensional Navier-Stokes Equation, we now obtain

$$
\rho_{\circ} \frac{\partial u}{\partial t}=\frac{\partial \sigma_{11}}{\partial x}=-\frac{\partial p}{\partial x}+\eta_{v} \frac{\partial^{2} u}{\partial x^{2}}+\eta_{p} \frac{\partial^{2} p}{\partial x \partial t}
$$

and the lossy wave equation becomes 


$$
\frac{\partial^{2} \rho}{\partial t^{2}}-\frac{\eta_{v}}{\rho_{0}} \frac{\partial^{3} \rho}{\partial x^{2} \partial t}=\frac{\partial^{2} p}{\partial x^{2}}-\eta_{p} \frac{\partial^{3} p}{\partial x^{2} \partial t}
$$

As before, solutions of the form (6a) and (6b) lead to the complex sound speed

$$
\underset{\tilde{p}}{\tilde{\rho}}=\frac{1}{c^{2}}=\frac{k^{2}-i \omega k^{2} \eta_{p}}{\omega^{2}-i \omega\left(\eta_{v} / \rho_{o}\right) k^{2}}=\frac{1}{c_{o}^{2}} \frac{1-i \omega \eta p}{1-i \omega \eta_{v} / \rho_{O} c_{o}^{2}}+O\left(\epsilon^{2}\right)
$$

Comparison of the complex sound speed derived from the volume viscosity [equation (22)] with that derived from the acoustical equation of state [equation ( 8 )] yields the volume viscosity parameters in terms of the acoustical relaxation parameters:

$$
\begin{aligned}
& \eta_{\mathrm{p}}=-\tau_{\mathrm{vs}} \\
& \eta_{\mathrm{v}}=-\rho_{\mathrm{o}} c_{\mathrm{o}}{ }^{2} \tau_{\mathrm{ps}}
\end{aligned}
$$

Both $\eta_{p}$ and $\eta_{v}$ are negative quantities in order to provide the proper phase relationships when equation (21) is applied to acoustic flow.

Volume viscosity and pressure relaxation cannot be modeled adequately via a single constitutive constant. The difficulty is due to the more fundamental character of pressure, in comparison to either volumetric viscous effects or shear stresses. Since pressure is accepted universally as a thermodynamic variable which can be used in the determination of other thermodynamic properties, it is not logical to force pressure to be related directly to the material rate of change of density, through the conservation of mass equation and a single volume viscosity coefficient. Furthermore, it is not logical to assume that pressure and density are determined uniquely for unsteady flows of simple fluids through an equilibrium equation of state, if those fluids possess internal degrees of freedom (which equilibrate at different rates). These relaxation effects are easily visualized in terms of the behavior of air when it is subjected to high frequency acoustic excitations. It is certainly evident that the temporal behavior of the density, produced by these acoustic oscillations, does not correspond to that produced by a reversible, quasi-static, cyclic equilibrium process. In fact, it is known that pressure must respond more rapidly to these propagating disturbances than density because the disturbances consist primarily of collisional 
exchanges of translational momentum, and pressure is a direct measure of translational momentum. Furthermore, since the nitrogen and oxygen molecules in air possess internal degrees of freedom that equilibrate more slowly than their translational counterparts, acoustically excited air is forced to exist in a regime where density and pressure are no longer in phase with each other, and temporal variations in internal molecular temperatures can be substantially different from their translational temperature histories. While it may be possible to ignore volume viscosity contributions completely, via stokes' hypothesis, it is not possible to ignore these phase shifts between density, temperature(s), and pressure that are manifest by these fundamental departures from thermodynamic equilibrium. If pressure equilibrium is enforced by an idealized equation of state, then the pressure is modified improperly via the traditional volume viscosity. It is certainly true that the pressure relaxation constant, introduced in our constitutive model, can be used along with volume viscosity; but, even further, it is quite possible that the pressure relaxation constant follows only the dynamic viscosity in importance as a constitutive constant.

\section{Multiple Relaxations}

The volumetric dissipation in many gases, notably air, is characterized not by one relaxation but by multiple relaxations occurring simultaneously. For the acoustical case Bauer shows that the effects of the individual relaxation processes upon the compressibility, as evidenced by the dispersion and absorption terms in equation (9), are additive [3]. The treatment here for generalized flow, then, is similarly based on the assumption that the effects of the individual relaxation processes are additive. A discussion of coupling among the relaxation processes appears at the end of this section.

We define $p_{R}(x)$ and $\rho_{R}(x)$ as the "relaxed" pressure and density, i.e. the solutions obtained without losses. Then we assume that the pressure $p(x)$ and density $\rho(x)$ are composed of the relaxed contribution plus the sum of the contributions from all the relaxation processes. If the gas is a mixture, then each contribution must be multiplied by the mole fraction $x_{k}$ of the relaxing component of the mixture: 


$$
\begin{aligned}
& p(x)=p_{R}(x)+\sum_{k} x_{k} \Delta p_{k}(x), \quad k=1,2, \ldots \\
& \rho(x)=\rho_{R}(x)+\sum_{k} x_{k} \Delta \rho_{k}(x)
\end{aligned}
$$

where $\Delta \mathrm{p}_{k}(\mathrm{x})$ and $\Delta \rho_{k}(\mathrm{x})$ are the changes in pressure and density due to the kth relaxation alone. These changes are not necessarily perturbations and can, in fact, be very large. If $p_{k}(x)$ and $\rho_{k}(x)$ are solutions to Navier-stokes Equation for the kth single relaxation process alone, using equation (19), then the changes are simply

$$
\begin{aligned}
\Delta p_{k}(x) & =p_{k}(x)-p_{R}(x) \\
\Delta \rho_{k}(x) & =\rho_{k}(x)-\rho_{R}(x)
\end{aligned}
$$

The above procedure is based on the condition that the presence of one relaxation process does not influence the effects due to another; in other words, the relaxations are decoupled. In reality this is not the case, for there are two types of coupling among the individual relaxations: gas-kinetic and nonlinear.

Gas-kinetic coupling arises from the fact that the reactants and products of molecular reactions can appear in more than one reaction. The relaxation times $\tau_{\mathrm{psk}}$ and $\tau_{\mathrm{vsk}}$ are no longer those of the decoupled kth relaxation alone. Rather they are determined from the solutions to a set of linear simultaneous equations involving all the coupled reactions. since the coupling is linear, the formulation expressed by equations (24)-(25) remains valid; only the values of $\tau_{\text {psk }}$ and $\tau_{\text {vsk }}$ have to be adjusted. For air this subject is treated in reference 5 .

The second type of coupling is due to the nonlinear nature of the convective operator $u \partial / \partial x$. When operating on equation (24), it will introduce mixed terms in the Navier-stokes Equation. Additional terms will be required in the series to account for the mixing. Generally, it may be expected that the volumetric losses and pressure and density changes are sufficiently small to permit equations $(24)-(25)$ to remain very accurate approximations for most gases. 


\section{An Example: Iinearly Accelerating Flow}

In order to examine the behavior of this constitutive model, it is convenient to examine a class of steady, one-dimensional flows. That class of flows includes flows through standing normal shock waves, but we are interested here in flows which are more typically encountered in the subsonic contraction sections of wind tunnels. If the velocity is given by $u=u(x)$, the material or total time derivative is given by

$$
\frac{D}{D t}=u \frac{d}{d x}
$$

and conservation of mass requires that

$$
\frac{d}{d x}(\rho u)=0
$$

Thus, if we assume that the density and velocity are prescribed at the coordinate origin--say $\rho_{0}$ and $U_{0}$--conservation of mass is equivalent to the requirement

$$
\rho(\mathrm{x}) \mathrm{u}(\mathrm{x})=\rho_{\mathrm{O}} \mathrm{U}_{\mathrm{O}}
$$

Conservation of linear momentum can be written

$$
\rho u \frac{d u}{d x}=\frac{d}{d x}\left(\rho_{O} U_{O} u\right)=\frac{d o}{11} d x
$$

and if we assume that $\sigma_{11}(0)=-p_{0}$, equation (28) can be integrated to yield

$$
\sigma_{11}(x)=-p_{0}-\rho_{0} U_{0}\left[U_{0}-u(x)\right]
$$

which is a kinematical relationship not affected directly by the constitutive model.

The constitutive model can be examined via the kinematical equation for normal stress. That is, $\sigma_{11}$ must satisfy the relationship

$$
\sigma_{11}(x)=-p(x)+\eta_{p} u \frac{d p}{d x}+\eta_{v} \frac{d u}{d x}=-p_{0}-\rho_{O} U_{o}\left(U_{0}-u\right)
$$

Before proceeding to the particular flow case, it is useful to distinguish between "lossless" pressure variations (where $\eta_{p}$, $\eta_{v}$ ' and $\mu$ are taken to be zero) and "lossy" effects. That is, we 
define $\Delta \overline{\mathbf{p}}(\mathrm{x})$ :

$$
\Delta \bar{p}(x)=\frac{p(x)-p_{0}-\rho_{o} U_{o}\left(U_{0}-u\right)}{\rho_{O} U_{0}{ }^{2}}
$$

and introduce the reduced and dimensionless variables

$$
\begin{aligned}
& \overline{\mathrm{u}}=\mathrm{u} / \mathrm{U}_{0} \\
& \overline{\mathrm{x}}=\mathrm{x} / \mathrm{L} \\
& \mathrm{T}=-\eta_{\mathrm{p}} \mathrm{U}_{\mathrm{O}} / \mathrm{L}=\mathrm{Mc_{O } \tau} \mathrm{vs} / \mathrm{L} \\
& \mathrm{R}_{\mathrm{e}}=\rho_{\mathrm{O}} \mathrm{U}_{\mathrm{O}} \mathrm{L} / \mu=\rho_{\mathrm{O}} \mathrm{Mc_{0 }} \mathrm{L} / \mu \\
& \kappa=-\eta_{\mathrm{v}} / \mu=\rho_{O} c_{0}{ }^{2} \tau_{\mathrm{ps}} / \mu
\end{aligned}
$$

Then, our constitutive model must satisfy

$$
\Delta \bar{p}=-T \bar{u}(d \Delta \bar{p} / d \bar{x})+\left(T \bar{u}-\kappa / R_{e}\right)(d \bar{u} / d \bar{x})
$$

For simplicity, we consider linearly accelerating flow systems, where:

$$
\bar{u}(\bar{x})=1+\bar{x}
$$

We note that in the case of this "contoured" wind tunnel contraction flow, the characteristic length $L$ is related to the length of the contraction section $\mathrm{L}_{\max }$ and the exit velocity $\mathrm{U}_{e}$ by:

$$
\mathrm{L}=\frac{\mathrm{L}_{\max } \mathrm{U}_{0}}{\mathrm{U}_{e}-\mathrm{U}_{0}} \text {, with } 0 \leq x \leq \frac{\mathrm{U}_{e}-\mathrm{U}_{0}}{\mathrm{U}_{e}}
$$

Here the lossless or "relaxed" pressure would decrease linearly (from $p_{0}$ ) with $\bar{x}$ :

$$
\overline{\mathbf{p}}_{\mathrm{R}}=\overline{\mathbf{p}}_{\mathrm{O}}-\overline{\mathrm{x}}
$$

where the overbar indicates a reduced pressure

$$
\overline{\mathbf{p}}=\mathrm{p} / \rho_{\mathrm{O}} \mathrm{U}_{\mathrm{O}}{ }^{2}
$$

The reduced lossy pressure variation can be gotten by integrating equations (33) and (34), with $\Delta \overline{\mathbf{p}}(0)=0$, to get:

$$
\Delta \overline{\mathrm{p}}=\frac{\mathrm{T}}{\mathrm{T}+1} \overline{\mathrm{x}}+\left[\frac{\kappa}{\mathrm{R}_{e}}-\frac{\mathrm{T}}{\mathrm{T}+1}\right][(1+\overline{\mathrm{x}})-1 / \mathrm{T}-1]
$$


which yields the interesting relation that volume viscosity will cause $\Delta \bar{p}$ to be negative, since $(1+\bar{x})-1 / T-1$ is negative for $\bar{x}>0$; whereas the pressure lag contributes an additional effect which is positive rather than negative. Since $\Delta \bar{p}=-\kappa / R_{e}$, when $\mathrm{T}=0$, the pressure lag effect changes the lossy pressure behavior fundamentally, by eliminating the instantaneous and constant $\Delta \bar{p}$ contribution, and introducing a contribution which is initially zero and becomes positive.

The significant relaxing degrees of freedom in air are the vibration of $\mathrm{N}_{2}$, vibration of $\mathrm{O}_{2}$, rotation of $\mathrm{N}_{2}$ and $\mathrm{O}_{2}$ taken together, and translation of all constituents. For relatively long time scales $(>1 \mu s$ ) only the two vibrational relaxations need be considered. Applying equation (38) together with (24a) and designating $\mathrm{N}_{2}$ and $\mathrm{O}_{2}$ with subscripts " $\mathrm{N}$ " and " $\mathrm{X}$," we find for the reduced pressure:

$$
\begin{aligned}
\bar{p}(\bar{x})=\bar{p}_{0} & +x_{N}\left\{\frac{T_{N}}{T_{N}+1} \bar{x}+\left[\frac{\kappa_{N}}{R_{e}}-\frac{T_{N}}{T_{N}+1}\right]\left[(1+\bar{x})-1 / T_{N}-1\right]\right\} \\
& +x_{X}\left\{\frac{T_{X}}{T_{X}+1} \bar{x}+\left[\frac{{ } X}{R_{e}}-\frac{T_{X}}{T_{X}+1}\right]\left[(1+\bar{x})-1 / T_{X}-1\right]\right\}
\end{aligned}
$$

Values for the relaxation times and relaxation strengths for $\mathrm{N}_{2}$ and $\mathrm{O}_{2}$ are taken from reference 6 . Then equations $(12 \mathrm{a}, \mathrm{b})$ and $(23 \mathrm{a}, \mathrm{b})$ are used to find $\tau_{\mathrm{ps}}, \tau_{\mathrm{vs}}, \eta_{\mathrm{p}}$, and $\eta_{\mathrm{v}}$. These are listed for a temperature of $20^{\circ} \mathrm{C}$ and humidity values of 0 and 1 mole percent in table 1. Because of the small relaxation strengths of $\mathrm{N}_{2}$ and $\mathrm{O}_{2}$, both $\tau$ ps and $\tau_{\mathrm{vs}}$ are nearly equal to the experimentally determined relaxation time $\tau$ for each gas constituent.

Equation (39) is plotted in figures 3 and 4 for a temperature $T=20^{\circ} \mathrm{C}$, length scale $\mathrm{L}=1 \mathrm{~m}$, and absolute humidities $\mathrm{h}=0$ and 1 mole percent, respectively. The terms $T /(T+1)$ related to pressure relaxation lead to a positive pressure increment, while the terms $\kappa /$ Re related to the traditional volume viscosity lead to a negative pressure increment (or decrement). A net pressure decrement is possible only if the volume viscosity terms exceed the pressure relaxation terms, as occurs at subsonic Mach numbers. In fact, at a speed Mach 0.9 the decrement turns into an increment at a reduced distance $\bar{x} \approx 0.4-0.5$. At Mach 1 and higher the increment is positive over the whole range of reduced 
distance. Unlike the acoustical case, there do not appear to be readily discernible conditions for which the increment reaches a maximum.

\section{Conclusions}

The traditional volume viscosity cannot be given the same fundamental status as dynamic viscosity. Not only does the traditional volume viscosity fail to evolve naturally from kinetic theory, but we have presented explanations showing that the volume viscosity coefficient must represent multiple molecular relaxation processes, including volumetric dissipation, simultaneously when the conventional Newtonian-fluid model is used. Furthermore, because of the direct relationship between the divergence of the velocity vector and the particle rate of change of density, volume viscosity in effect forces normal stresses, including pressure, to be modeled in a manner which is inconsistent with the more rigorous requirements resulting from nonequilibrium acoustic equations of state. We have proposed that a pressure relaxation contribution be added directly to the constitutive model for normal stresses, in order to bring the constitutive model into better agreement with accepted nonequilibrium behavior.

Using accepted acoustical theory, we have estimated the values for the pressure relaxation coefficients and volume viscosities in pure nitrogen and oxygen at $20^{\circ} \mathrm{C}$. Those estimates indicate that the nitrogen contributions are nearly an order of magnitude larger than the oxygen contributions, but both coefficients are influenced strongly by humidity. Our estimates show that the volume viscosity is several million times larger than the dynamic viscosity for perfectly dry nitrogen, but the ratio of volume to dynamic viscosity decreases by a factor of 32 at moderately high humidity levels (at $20^{\circ} \mathrm{C}$ ). In addition, if pressure fluctuations are on the order of $1 \mathrm{~Pa} / \mathrm{s}$, the pressure relaxation contribution to normal stresses is nearly an order of magnitude larger than the volume viscosity contribution, when the modified constitutive model is employed.

The application to a simple problem in one-dimensional convective flow reveals that there are conditions for which the volumetric losses in air are negligible (low Mach number, moderate humidity), in agreement with the stokes' hypothesis, but other conditions for which they are substantial (high Mach number, low humidity). The latter case suggests caution to the 
advocates of the popular notion that computers will replace wind tunnels, for a computational algorithm is effective only to the extent that it incorporates the significant physical

interactions. A computation ignoring or misrepresenting volumetric losses may fail to predict effects which would be observed in wind tunnel tests.

The constitutive model proposed here should apply equally well to liquids, since the acoustical equation of state is the same as for gases [7]. The constitutive coefficients can be determined from acoustical relaxation data through equations $(23 a-b)$. Then the volumetric losses due to thermal relaxation [8], for example, can be computed for convective flow in liquids.

\section{References}

1. Hirschfelder, J. O.; Curtiss, C. F.; and Bird, R. B.: Molecular Theory of Gases and Liquids. John Wiley and Sons, Inc., 1954 , p. 480 .

2. Schlichting, H.: Boundary-Layer Theory. McGraw-Hill Book Company, 1987, p. 60 .

3. Bauer, H. J.: "Phenomenological Theory of the Relaxation Phenomena in Gases," Physical Acoustics IIA. W. P. Mason, editor, Academic Press, 1965, p. 65.

4. Kneser, H. O.: "Relaxation Processes in Gases," Physical Acoustics IIA. W. P. Mason, editor, Academic Press, 1965, p.141.

5. Zuckerwar, A. J.; and Miller, K. W.: "VibrationalVibrational coupling in Air at Low Humidities," J. Acoust. Soc. Am. 84, pp. 970-977, 1988.

6. ANSI S1.26-1978, "American National Standard Method for Calculation of the Absorption of Sound by the Atmosphere," Acoustical Society of America, New York, 1981.

7. Lamb, J.: "Thermal Relaxation in Liquids," Physical Acoustics IIA. W. P. Mason, editor, Academic Press, 1965, p. 218.

8. Nettleton, R. E.: "Thermodynamics of Viscoelasticity in Liquids," Phys. Fluids 2, pp. 256-263, 1959. 
Table 1. Volume Viscosity Parameters for $\mathrm{N}_{2}$ and $\mathrm{O}_{2}$ at $20^{\circ} \mathrm{C}$.

\begin{tabular}{|c|c|c|c|c|c|c|}
\hline \multicolumn{2}{|c|}{$\begin{array}{l}\text { Humidity, mole percent } \\
\text { Air constituent } \\
\text { Parameter }\end{array}$} & \multirow[t]{2}{*}{ Unit } & $\mathrm{N}_{2}$ & $\mathrm{O}_{2}$ & $\mathrm{~N}_{2}$ & $\mathrm{O}_{2}$ \\
\hline Relaxation strength & $\epsilon$ & & 0.00016 & 0.0032 & 0.00016 & 0.0032 \\
\hline \multirow[t]{3}{*}{ Relaxation times } & $\tau$ & $\mu s$ & 17700 & 6630 & 551 & 5.37 \\
\hline & ${ }^{\tau}$ ps & $\mu \mathbf{s}$ & 17700 & 6640 & 551 & 5.38 \\
\hline & $\tau_{\mathrm{vs}}$ & $\mu \mathbf{s}$ & 17700 & 6620 & 551 & 5.36 \\
\hline \multirow{2}{*}{$\begin{array}{l}\text { Volume viscosity } \\
\text { coefficients }\end{array}$} & $\eta_{\mathrm{p}}$ & $\mu \mathbf{s}$ & -17700 & -6620 & -551 & -5.36 \\
\hline & $\eta_{\mathrm{V}}$ & $\mathrm{Pa} . \mathrm{s}$ & -2512.6 & -943.6 & -78.24 & -0.764 \\
\hline Mole fraction & $\mathbf{x}$ & & 0.79 & 0.21 & 0.79 & 0.21 \\
\hline
\end{tabular}



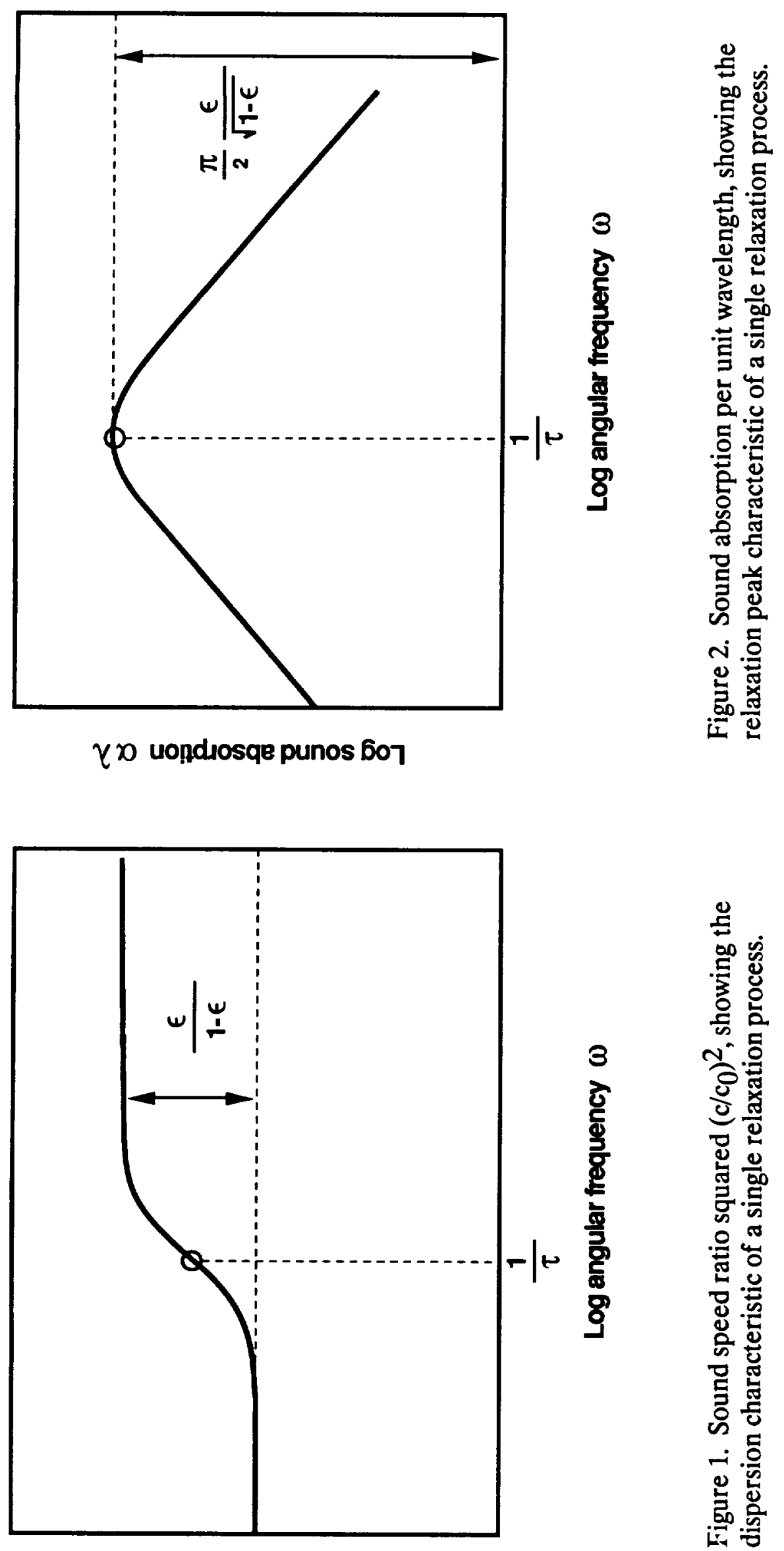

${ }_{2}$ (0\%/O) pasenbs ones poods punos 


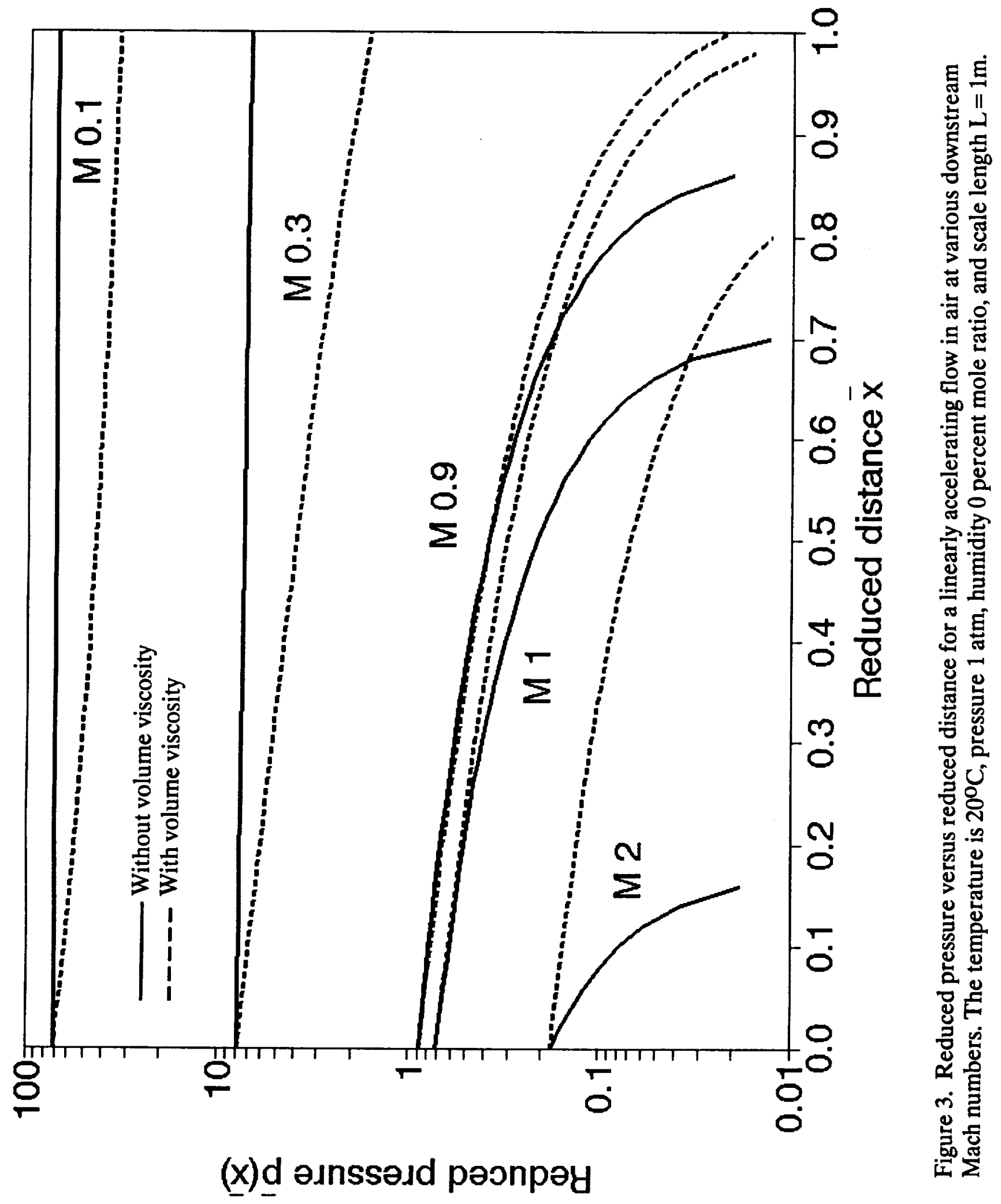




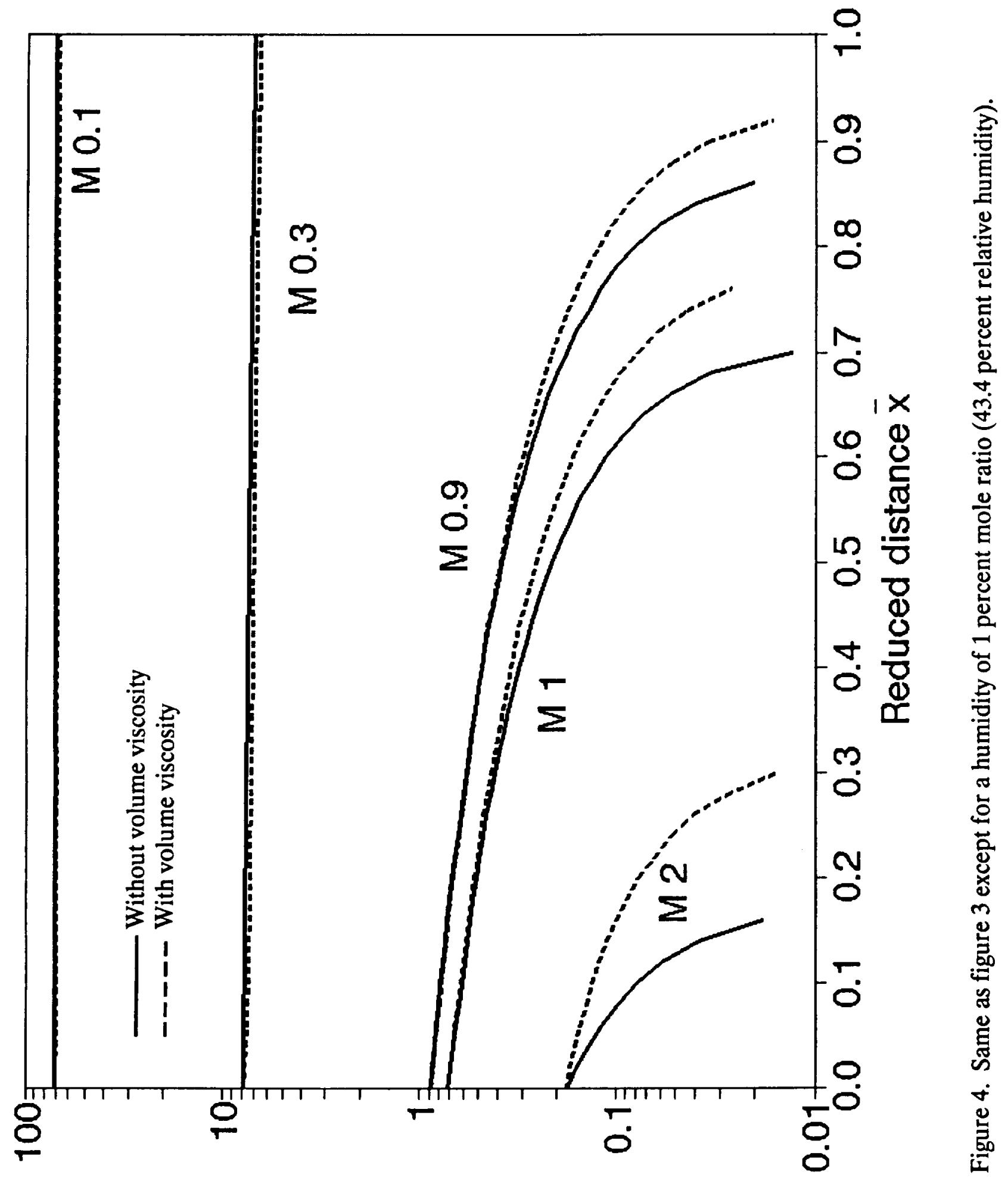

$(x) d$ əınssəנd pəonpəy 


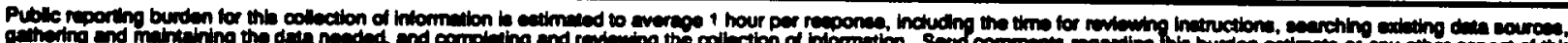

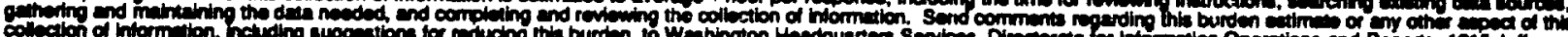

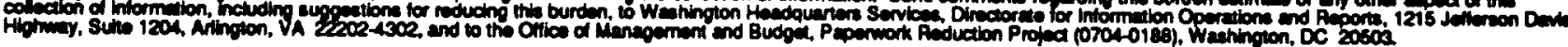

\begin{tabular}{|l|l|l|}
\hline 1. ACENCY USE ONLY (Lavo blank) & $\begin{array}{l}\text { 2. AEPOAT DATE } \\
\text { March } 1994\end{array}$ & $\begin{array}{l}\text { 3. AEPORT TYPE AND DATES COVEAED } \\
\text { Technical Memorandum }\end{array}$ \\
\hline
\end{tabular}

4. TILL AND SUBTTILE

New Constitutive Equation for the Volume Viscosity in Fluids
5. FUNDINO NUMBERS

505-59-54-02

6. AUTHOR(S)

Allan J. Zuckerwar and Robert L. Ash

7. PEAFORMINO ORCANIZATION NAME(S) AND ADDRESSKES)

NASA Langley Research Center

Hampton, VA 23681-0001
Q. PERFORMINO OROANIZTTLO REPOAT NUMBER
9. अPONSOAING / MONITORING AGENCY NAME(S) AND ADDRESS(ES)

National Aeronautics and Space Administration

Washington, DC 20546-0001
10. SPONSOALA / MONTORING ACENCY REPOAT MUMAER

NASA TM-109104.

11. SUPPLEMENTARY NOTES

Zuckerwar: Langley Research Center, Hampton, VA; Ash: Old Dominion University, Norfolk, VA. Presented at 127th Meeting of the Acoustical Society of America, Cambridge, MA, June 6-10, 1994.

12a. DISTRIBUTION / AVALABILTY STATEMENT

Unclassified-Unlimited

Subject Category 34

\section{ABSTALCT (Maximum 200 wordd)}

The traditional volume viscosity, Stokes' hypothesis, and acoustical relaxation are reviewed. The lossy Navier-Stokes Equation is applied to periodic (acoustic) flow, and it is shown that the traditional volume viscosity leads to a result which contradicts that describing acoustical relaxation. It is demonstrated that the addition of a second volume viscosity term to the constitutive equation, to account for pressure relaxation, resolves the conflict and leads to a direct correspondence between the volume viscosity parameters and the acoustical relaxation parameters. The representation of volume viscosity is formulated for the case of multiple relaxations, as occur in air. Finally, an application of the new constitutive equation to a simple convective compressible flow, namely a linearly accelerating flow, demonstrates the impact of volume viscosity upon the flow and the physical conditions for which it is important.

\section{SUBJECT TERIMS}

Volume viscosity; Molecular relaxation; Constitutive equation

15. MUWBEA OFPAGES

20

16. PAICE CODE

$\mathrm{AO} 3$

\begin{tabular}{l|l|}
$\begin{array}{l}\text { 17. SECURTY CLASSIFICATION } \\
\text { OF REPOAT } \\
\text { Unclassified }\end{array}$ & $\begin{array}{c}\text { 18. SECUATYY CLASSIFICATION } \\
\text { OF THIS PAGE } \\
\text { Unclassified }\end{array}$ \\
\hline
\end{tabular}

NSN 7540-01-280-5500

19. SECUATY CLASSIFICATION
OF ABSTAACT
Unclassified

20. LIITATION OF ABSTRACT

Standard Forws 209 (Rov. 2-99) Promatbed by ANSI Sid. 20018 293-102 